\title{
Existence and exponential stability of the unique positive almost periodic solution for the Lasota-Wazewska difference model
}

\section{Zhijian Yao*}

\section{"Correspondence:}

zhijianyao@126.com

Department of Mathematics and

Physics, Anhui University of

Architecture, Hefei, Anhui 230601, China

\begin{abstract}
In this paper, a discrete Lasota-Wazewska model is studied. By using the fixed point theorem of decreasing operator, we obtain sufficient conditions for the existence of a unique positive almost periodic solution. Particularly, we give iterative sequence which converges to the positive almost periodic solution. Moreover, we investigate exponential stability of the positive almost periodic solution by the Lyapunov functional.
\end{abstract}

MSC: 39A24; 39A30

Keywords: discrete Lasota-Wazewska model; almost periodic solution; uniqueness; exponential stability; fixed point theorem of decreasing operator

\section{Introduction}

Biological dynamic models are very important and hot research topics. In 1976, Wazewska-Czyzewska and Lasota [1] investigated the Lasota-Wazewska model

$$
x^{\prime}(t)=-a x(t)+b e^{-p x(t-\tau)}
$$

which described the survival of red blood cells in animals. Kulenovic and Ladas [2] investigated the oscillation and global attractivity of the above model (1.1). Moreover, the model (1.1) and some generalized models have been investigated by many authors; see Graef et al. [3], Kulenovic et al. [4], Xu and Li [5], Jiang [6], Li and Wang [7].

The assumption that the environment is constant is rarely the case in real life. When the environmental fluctuation is taken into account, a model must be nonautonomous. Due to the various seasonal effects of the environmental factors in a real life situation (e.g., seasonal effects of weather, food supplies, mating habits, harvesting, etc.), it is rational and practical to study the biological system with periodic coefficients or almost periodic coefficients. Many authors [6,7] have studied nonautonomous differential equations with periodic coefficients of the above model (1.1).

Though most models are described with differential equations, the discrete-time models governed by difference equations are more appropriate than the continuous ones when the size of the population is rarely small or the population has non-overlapping generations. It is also known that the discrete models can provide more efficient computational methods

C2014 Yao; licensee Springer. This is an Open Access article distributed under the terms of the Creative Commons Attribution License (http://creativecommons.org/licenses/by/2.0), which permits unrestricted use, distribution, and reproduction in any medium, provided the original work is properly cited. 
for numerical simulations [8-10]. However, the studies in the past [2-7] were concerned with the continuous case of the above model (1.1).

To our knowledge, studies on the uniqueness and exponential stability of positive almost periodic solutions for discrete models are scarce.

Motivated by the above facts, in this paper, we investigate the following LasotaWazewska difference equation:

$$
\Delta x(k)=-a(k) x(k)+b(k) e^{-\beta(k) x(k-\tau)},
$$

where $\Delta x(k)=x(k+1)-x(k), a(k): Z \rightarrow(0,1), b(k): Z \rightarrow(0,+\infty), \beta(k): Z \rightarrow(0,+\infty)$, $a(k), b(k), \beta(k)$ are bounded almost periodic functions, $\tau \in Z^{+}$. Due to biological reasons, we restrict our attention to positive solutions of equation (1.2). The initial condition of equation (1.2) is $x(k)=\phi(k)>0$ for $-\tau \leq k \leq 0$.

In the study of biological systems, an important ecological problem is concerned with the existence of positive periodic solutions or positive almost periodic solutions. Recently, many authors investigated the existence of positive periodic solutions by using the Krasnoselskii cone fixed point theorem and Mawhin's coincidence degree theory [6, 7, 11-13]. Most of the past studies are concerned with the existence of at least one positive periodic solution $[6,11,12,14]$.

Almost periodicity is more practical and more close to the reality in biological systems $[15,16]$, the recent contributions such as the almost periodic solutions of delay and impulsive differential equations [17-20] have appeared.

However, few papers study the existence and exponential stability of unique positive almost periodic solutions for discrete models. For the existence and uniqueness of a positive almost periodic solution, the method used in most of the past studies is the contraction mapping fixed point theorem.

In this paper, different from the past studies, we aim to obtain sufficient conditions that guarantee the existence of a unique positive almost periodic solution of discrete model (1.2) by using the fixed point theorem of decreasing operator. Particularly, we give an iterative sequence which converges to the positive almost periodic solution. We also obtain sufficient conditions for the exponential stability of the unique positive almost periodic solution by means of the Lyapunov functional. The results of this paper are new and more valuable in applications, which complement the previously obtained results in [2-7].

\section{Preliminaries}

For any bounded sequence $\{f(k)\}$, we define $\bar{f}=\sup _{k \in Z} f(k), f=\inf _{k \in Z} f(k)$.

For equation (1.2), we assume that the bounded almost periodic sequences $\{a(k)\},\{b(k)\}$, $\{\beta(k)\}$ satisfy $0<\underline{a} \leq a(k) \leq \bar{a}<1,0<\underline{b} \leq b(k) \leq \bar{b}, 0<\underline{\beta} \leq \beta(k) \leq \bar{\beta}$.

Definition 1 [21] A sequence $x(k): Z \rightarrow R$ is called an almost periodic sequence if the $\varepsilon$-translation set $E\{\varepsilon, x\}=\{\delta \in Z:|x(k+\delta)-x(k)|<\varepsilon, \forall k \in Z\}$ is a relatively dense set in $Z$ for all $\varepsilon>0$; that is, for any $\varepsilon>0$, there exists a constant $l(\varepsilon)>0$ such that each interval of length $l(\varepsilon)$ contains a number $\delta \in E\{\varepsilon, x\}$ such that $|x(k+\delta)-x(k)|<\varepsilon$ for all $k \in Z$.

$\delta$ is called the $\varepsilon$-translation number of $x(k)$.

Definition 2 Let $X$ be a Banach space and $P$ be a closed, nonempty subset of $X$. $P$ is called a cone if 
(i) $x \in P, \lambda \geq 0$ implies $\lambda x \in P$;

(ii) $x \in P,-x \in P$ implies $x=\theta$.

Every cone $P \subset X$ induces an ordering in $X$, we define ' $\leq$ ' with respect to $P$ by $x \leq y$ if and only if $y-x \in P$.

Definition 3 A cone $P$ of $X$ is called a normal cone if there exists a positive constant $\sigma$ such that $\|x+y\| \geq \sigma$ for any $x, y \in P,\|x\|=\|y\|=1$.

Definition 4 Let $P$ be a cone of $X$ and $A: P \rightarrow P$ be an operator. $A$ is called decreasing if $\theta \leq x \leq y$ implies $A x \geq A y$.

The following fixed point theorem of decreasing operator (see [22]) is an important tool in our proofs.

\section{Lemma 1 [22] Suppose that}

(i) $P$ is a normal cone of the Banach space $X$, the operator $A: P \rightarrow P$ is decreasing;

(ii) $A \theta>\theta, A^{2} \theta \geq \varepsilon_{0} A \theta$, where $\varepsilon_{0}>0$

(iii) For $\forall 0<a<c<1$, there exists $\eta=\eta(a, c)>0$ such that

$$
A(\lambda x) \leq[\lambda(1+\eta)]^{-1} A x \quad \text { for } \forall a \leq \lambda \leq c \text { and } \theta<x \leq A \theta .
$$

Then $A$ has a unique positive fixed point $x^{*}>\theta$. Moreover, $\left\|x_{k}-x^{*}\right\| \rightarrow 0(k \rightarrow \infty)$, where $x_{k}=A x_{k-1}(k=1,2, \ldots)$ for any initial $x_{0} \in P$.

In this paper, we will use the above Lemma 1 to investigate the existence of a unique positive almost periodic solution of model (1.2).

Remark In Lemma 1, the operator $A$ does not need continuity and compactness.

Lemma 2 Every solution $x(k)$ of equation (1.2) is positive.

Proof Let $x(k)$ be the solution of equation (1.2), then we have

$$
x(k+1)-[1-a(k)] x(k)=b(k) e^{-\beta(k) x(k-\tau)} .
$$

Hence we get

$$
\begin{aligned}
\left(\mathrm{E}_{1}\right) & x(k)-[1-a(k-1)] x(k-1)=b(k-1) e^{-\beta(k-1) x(k-1-\tau)}, \\
\left(\mathrm{E}_{2}\right) & x(k-1)-[1-a(k-2)] x(k-2)=b(k-2) e^{-\beta(k-2) x(k-2-\tau),} \\
\left(\mathrm{E}_{3}\right) & x(k-2)-[1-a(k-3)] x(k-3)=b(k-3) e^{-\beta(k-3) x(k-3-\tau)}, \\
\ldots & \\
\left(\mathrm{E}_{k-1}\right) & x(2)-[1-a(1)] x(1)=b(1) e^{-\beta(1) x(1-\tau)}, \\
\left(\mathrm{E}_{k}\right) & x(1)-[1-a(0)] x(0)=b(0) e^{-\beta(0) x(0-\tau)} .
\end{aligned}
$$


Multiplying two sides of $\left(\mathrm{E}_{2}\right),\left(\mathrm{E}_{3}\right), \ldots,\left(\mathrm{E}_{k-1}\right),\left(\mathrm{E}_{k}\right)$ by $1-a(k-1),[1-a(k-1)][1-a(k-2)]$, $\ldots,[1-a(k-1)][1-a(k-2)] \cdots[1-a(2)],[1-a(k-1)][1-a(k-2)] \cdots[1-a(2)][1-a(1)]$, respectively, we get

$\left(\mathrm{E}_{2}^{\prime}\right) \quad[1-a(k-1)] x(k-1)-[1-a(k-1)][1-a(k-2)] x(k-2)$

$$
=b(k-2) e^{-\beta(k-2) x(k-2-\tau)}[1-a(k-1)],
$$

$\left(\mathrm{E}_{3}^{\prime}\right) \quad[1-a(k-1)][1-a(k-2)] x(k-2)$

$$
\begin{aligned}
& -[1-a(k-1)][1-a(k-2)][1-a(k-3)] x(k-3) \\
= & b(k-3) e^{-\beta(k-3) x(k-3-\tau)}[1-a(k-1)][1-a(k-2)],
\end{aligned}
$$

$\left(\mathrm{E}_{k-1}^{\prime}\right) \quad[1-a(k-1)][1-a(k-2)] \cdots[1-a(2)] x(2)$

$$
\begin{aligned}
& -[1-a(k-1)][1-a(k-2)] \cdots[1-a(2)][1-a(1)] x(1) \\
= & b(1) e^{-\beta(1) x(1-\tau)}[1-a(k-1)][1-a(k-2)] \cdots[1-a(2)],
\end{aligned}
$$

$\left(\mathrm{E}_{k}^{\prime}\right) \quad[1-a(k-1)][1-a(k-2)] \cdots[1-a(2)][1-a(1)] x(1)$

$$
\begin{aligned}
& -[1-a(k-1)][1-a(k-2)] \cdots[1-a(2)][1-a(1)][1-a(0)] x(0) \\
= & b(0) e^{-\beta(0) x(0-\tau)}[1-a(k-1)][1-a(k-2)] \cdots[1-a(2)][1-a(1)] .
\end{aligned}
$$

Summing $\left(\mathrm{E}_{1}\right),\left(\mathrm{E}_{2}^{\prime}\right),\left(\mathrm{E}_{3}^{\prime}\right), \ldots,\left(\mathrm{E}_{k-1}^{\prime}\right),\left(\mathrm{E}_{k}^{\prime}\right)$, we get

$$
\begin{gathered}
x(k)-[1-a(k-1)][1-a(k-2)] \cdots[1-a(2)][1-a(1)][1-a(0)] x(0) \\
=b(k-1) e^{-\beta(k-1) x(k-1-\tau)}+\sum_{s=0}^{k-2}\left(b(s) e^{-\beta(s) x(s-\tau)} \prod_{i=s+1}^{k-1}(1-a(i))\right) .
\end{gathered}
$$

That is,

$$
x(k)=x(0) \prod_{s=0}^{k-1}(1-a(s))+b(k-1) e^{-\beta(k-1) x(k-1-\tau)}+\sum_{s=0}^{k-2}\left(b(s) e^{-\beta(s) x(s-\tau)} \prod_{i=s+1}^{k-1}(1-a(i))\right) .
$$

Since $x(k)=\phi(k)>0$ for $-\tau \leq k \leq 0$, we can deduce that $x(1)>0, x(2)>0, \ldots$, and $x(k)>0$ for all $k \in Z^{+}$. The proof is complete.

Let $X=\{x(k) \mid x(k): Z \rightarrow R, x(k)$ be an almost periodic function $\}$. For $x \in X$, we define $\|x\|=\sup _{k \in Z}|x(k)|$, then $X$ is a Banach space.

It is easy to verify that $x(k)$ is the solution of equation (1.2) if and only if $x(k)$ is the solution of the following equation:

$$
x(k)=b(k-1) e^{-\beta(k-1) x(k-1-\tau)}+\sum_{s=-\infty}^{k-2}\left(b(s) e^{-\beta(s) x(s-\tau)} \prod_{i=s+1}^{k-1}(1-a(i))\right) .
$$


We define the operator $A: X \rightarrow X$

$$
(A x)(k)=b(k-1) e^{-\beta(k-1) x(k-1-\tau)}+\sum_{s=-\infty}^{k-2}\left(b(s) e^{-\beta(s) x(s-\tau)} \prod_{i=s+1}^{k-1}(1-a(i))\right) .
$$

Obviously, $x(k) \in X$ is the almost periodic solution of equation (1.2) if and only if $x$ is the fixed point of the operator $A$.

Let

$$
W(k, s)=\prod_{i=s+1}^{k-1}(1-a(i)), \quad-\infty<s<k-1, s \in Z .
$$

Then

$$
W(k, s)=\prod_{i=s+1}^{k-1}(1-a(i)) \leq \prod_{i=s+1}^{k-1}(1-\underline{a})=(1-\underline{a})^{k-1-s} \leq 1-\underline{a}<1 .
$$

Define

$$
m=\inf _{\substack{k \in Z, s \in Z \\-\infty<s<k-1}} W(k, s)
$$

then $m<1$.

For $x \in X$, we define

$$
\begin{aligned}
& J_{x}=\sup _{k \in Z}\left\{\sum_{s=-\infty}^{k-1} e^{-\underline{\beta} x(s-\tau)}\right\}, \quad Q_{x}=\inf _{k \in Z}\left\{\sum_{s=-\infty}^{k-1} e^{-\bar{\beta} x(s-\tau)}\right\}, \\
& \bar{J}=\sup _{x \in X}\left\{J_{x}\right\}, \quad Q=\inf _{x \in X}\left\{Q_{x}\right\} .
\end{aligned}
$$

Define the cone

$$
\Omega=\{x \mid x \in X, x(k) \geq 0, x(k) \geq \gamma\|x\|\},
$$

here $\gamma=\frac{m \underline{b} \underline{\underline{Q}}}{\overline{\bar{J}}}<1$.

Lemma $3 A \Omega \subset \Omega$.

Proof For $\forall x \in \Omega$,

$$
\begin{aligned}
\|A x\| & =\sup _{k \in Z}|(A x)(k)|=\sup _{k \in Z}\left|b(k-1) e^{-\beta(k-1) x(k-1-\tau)}+\sum_{s=-\infty}^{k-2}\left(b(s) e^{-\beta(s) x(s-\tau)} W(k, s)\right)\right| \\
& \leq \sup _{k \in Z}\left\{b(k-1) e^{-\beta(k-1) x(k-1-\tau)}+(1-\underline{a}) \sum_{s=-\infty}^{k-2}\left(b(s) e^{-\beta(s) x(s-\tau)}\right)\right\} \\
& \leq \sup _{k \in Z}\left\{b(k-1) e^{-\beta(k-1) x(k-1-\tau)}+\sum_{s=-\infty}^{k-2}\left(b(s) e^{-\beta(s) x(s-\tau)}\right)\right\}
\end{aligned}
$$




$$
\begin{aligned}
& \leq \sup _{k \in Z}\left\{\bar{b} e^{-\underline{\beta} x(k-1-\tau)}+\bar{b} \sum_{s=-\infty}^{k-2} e^{-\underline{\beta} x(s-\tau)}\right\}=\sup _{k \in Z}\left\{\bar{b} \sum_{s=-\infty}^{k-1} e^{-\underline{\beta} x(s-\tau)}\right\} \\
& =\bar{b} \sup _{k \in Z}\left\{\sum_{s=-\infty}^{k-1} e^{-\underline{\beta} x(s-\tau)}\right\}=\bar{b} J_{x} \leq \bar{b} \bar{J} .
\end{aligned}
$$

On the other hand,

$$
\begin{aligned}
(A x)(k) & =b(k-1) e^{-\beta(k-1) x(k-1-\tau)}+\sum_{s=-\infty}^{k-2}\left(b(s) e^{-\beta(s) x(s-\tau)} W(k, s)\right) \\
& \geq \underline{b} e^{-\bar{\beta} x(k-1-\tau)}+m \underline{b} \sum_{s=-\infty}^{k-2} e^{-\bar{\beta} x(s-\tau)}>m \underline{b} e^{-\bar{\beta} x(k-1-\tau)}+m \underline{b} \sum_{s=-\infty}^{k-2} e^{-\bar{\beta} x(s-\tau)} \\
& =m \underline{b} \sum_{s=-\infty}^{k-1} e^{-\bar{\beta} x(s-\tau)} \geq m \underline{b} Q_{x} \geq m \underline{b} \underline{Q} .
\end{aligned}
$$

Hence

$$
(A x)(k) \geq m \underline{b} \underline{Q}=\frac{m \underline{b} \underline{Q}}{\bar{J}} \bar{J} \geq \frac{m \underline{b} \underline{Q}}{\bar{J}} \frac{\|A x\|}{\bar{b}}=\gamma\|A x\| .
$$

Thus $A x \in \Omega$, so we have $A \Omega \subset \Omega$. The proof is complete.

\section{Existence and uniqueness of a positive almost periodic solution}

Let $B^{+}=\sup _{k \in Z} \sum_{s=-\infty}^{k} b(s), B^{-}=\inf _{k \in Z} \sum_{s=-\infty}^{k} b(s)$.

Theorem 1 Assume that $\bar{\beta}\left[\bar{b}+(1-\underline{a}) B^{+}\right] \leq 1$, then equation (1.2) has a unique almost periodic positive solution $x^{*}(k)$. Moreover, $\left\|x_{k}-x^{*}\right\| \rightarrow 0(k \rightarrow \infty)$, where $x_{k}=A x_{k-1}(k=$ $1,2, \ldots)$ for any initial $x_{0} \in \Omega$.

Proof It is clear that $\Omega$ is a normal cone, $A: \Omega \rightarrow \Omega$ is a decreasing operator.

Now, we will show that condition (ii) of Lemma 1 is satisfied.

$$
\begin{aligned}
\bar{b}+(1-\underline{a}) B^{+} & \geq \bar{b}+(1-\underline{a}) \sum_{s=-\infty}^{k-2} b(s) \geq(A \theta)(k)=b(k-1)+\sum_{s=-\infty}^{k-2}(b(s) W(k, s)) \\
& \geq b(k-1)+m \sum_{s=-\infty}^{k-2} b(s)>0,
\end{aligned}
$$

which implies $A \theta>\theta$.

Again, we have

$$
\begin{aligned}
\left(A^{2} \theta\right)(k) & =b(k-1) e^{-\beta(k-1)(A \theta)(k-1-\tau)}+\sum_{s=-\infty}^{k-2}\left(b(s) e^{-\beta(s)(A \theta)(s-\tau)} \prod_{i=s+1}^{k-1}(1-a(i))\right) \\
& \geq b(k-1) e^{-\beta(k-1)\left[\bar{b}+(1-\underline{a}) B^{+}\right]}+\sum_{s=-\infty}^{k-2}\left(b(s) e^{-\beta(s)\left[\bar{b}+(1-\underline{a}) B^{+}\right]} \prod_{i=s+1}^{k-1}(1-a(i))\right)
\end{aligned}
$$




$$
\begin{aligned}
& \geq b(k-1) e^{-\bar{\beta}\left[\bar{b}+(1-\underline{a}) B^{+}\right]}+\sum_{s=-\infty}^{k-2}\left(b(s) e^{-\bar{\beta}\left[\bar{b}+(1-\underline{a}) B^{+}\right]} \prod_{i=s+1}^{k-1}(1-a(i))\right) \\
& =e^{-\bar{\beta}\left[\bar{b}+(1-\underline{a}) B^{+}\right]}\left[b(k-1)+\sum_{s=-\infty}^{k-2}\left(b(s) \prod_{i=s+1}^{k-1}(1-a(i))\right)\right]=e^{-\bar{\beta}\left[\bar{b}+(1-\underline{a}) B^{+}\right]}(A \theta)(k) \\
& =\varepsilon_{0}(A \theta)(k),
\end{aligned}
$$

which implies $A^{2} \theta \geq \varepsilon_{0} A \theta$, here $\varepsilon_{0}=e^{-\bar{\beta}\left[\bar{b}+(1-\underline{a}) B^{+}\right]}$.

Finally, we show that condition (iii) of Lemma 1 is satisfied.

Let $\forall 0<a<c<1$, for $\forall a \leq \lambda \leq c$ and $\theta<x \leq A \theta$, we have $0<\|x\| \leq\|A \theta\| \leq \bar{b}+(1-\underline{a}) B^{+}$.

$$
\begin{aligned}
A(\lambda x)(k)= & b(k-1) e^{-\beta(k-1) \lambda x(k-1-\tau)}+\sum_{s=-\infty}^{k-2}\left(b(s) e^{-\beta(s) \lambda x(s-\tau)} \prod_{i=s+1}^{k-1}(1-a(i))\right) \\
= & b(k-1) e^{-\beta(k-1) x(k-1-\tau)} e^{(1-\lambda) \beta(k-1) x(k-1-\tau)} \\
& +\sum_{s=-\infty}^{k-2}\left(b(s) e^{-\beta(s) x(s-\tau)} e^{(1-\lambda) \beta(s) x(s-\tau)} W(k, s)\right) \\
\leq & b(k-1) e^{-\beta(k-1) x(k-1-\tau)} e^{(1-\lambda) \bar{\beta}\left[\bar{b}+(1-\underline{a}) B^{+}\right]} \\
& +\sum_{s=-\infty}^{k-2}\left(b(s) e^{-\beta(s) x(s-\tau)} e^{(1-\lambda) \bar{\beta}\left[\bar{b}+(1-\underline{a}) B^{+}\right]} W(k, s)\right) \\
= & \frac{1}{\lambda} \cdot \lambda e^{(1-\lambda) \bar{\beta}\left[\bar{b}+(1-\underline{a}) B^{+}\right]}\left[b(k-1) e^{-\beta(k-1) x(k-1-\tau)}+\sum_{s=-\infty}^{k-2}\left(b(s) e^{-\beta(s) x(s-\tau)} W(k, s)\right)\right] .
\end{aligned}
$$

Let $f(x)=x e^{(1-x) \bar{\beta}\left[\bar{b}+(1-\underline{a}) B^{+}\right]}$, we have

$$
\begin{aligned}
f^{\prime}(x) & =e^{(1-x) \bar{\beta}\left[\bar{b}+(1-\underline{a}) B^{+}\right]}-x \bar{\beta}\left[\bar{b}+(1-\underline{a}) B^{+}\right] e^{(1-x) \bar{\beta}\left[\bar{b}+(1-\underline{a}) B^{+}\right]} \\
& =\left(1-x \bar{\beta}\left[\bar{b}+(1-\underline{a}) B^{+}\right]\right) e^{(1-x) \bar{\beta}\left[\bar{b}+(1-\underline{a}) B^{+}\right]}
\end{aligned}
$$

Since $\bar{\beta}\left[\bar{b}+(1-\underline{a}) B^{+}\right] \leq 1$, we know $f^{\prime}(x)>0$ for $0<x<1$, so we have

$$
0=f(0)<f(a) \leq f(\lambda) \leq f(c)<f(1)=1 .
$$

Hence we get

$$
\begin{aligned}
A(\lambda x)(k) & \leq \frac{1}{\lambda} \cdot f(\lambda)\left[b(k-1) e^{-\beta(k-1) x(k-1-\tau)}+\sum_{s=-\infty}^{k-2}\left(b(s) e^{-\beta(s) x(s-\tau)} W(k, s)\right)\right] \\
& \leq \frac{1}{\lambda} \cdot f(c)\left[b(k-1) e^{-\beta(k-1) x(k-1-\tau)}+\sum_{s=-\infty}^{k-2}\left(b(s) e^{-\beta(s) x(s-\tau)} W(k, s)\right)\right] \\
& =\frac{1}{\lambda} \cdot f(c)(A x)(k)=\frac{1}{\lambda} \cdot \frac{1}{1+\eta(c)}(A x)(k),
\end{aligned}
$$

here $\eta=\eta(c)=\frac{1}{f(c)}-1>0$. 
By Lemma 1, we know that the operator $A$ has a unique positive fixed point $x^{*}>\theta, \| x_{k}-$ $x^{*} \| \rightarrow 0(k \rightarrow \infty), x_{k}=A x_{k-1}(k=1,2, \ldots)$ for any initial $x_{0} \in \Omega$. The proof of Theorem 1 is complete.

Remark 1 Theorem 1 of this paper not only gives sufficient conditions for the existence of a unique positive almost periodic solution, but also gives the iterative sequence $\left\{x_{k}\right\}$ which converges to the positive almost periodic solution $x^{*}(k)$.

Remark 2 From the above proof, we have

$$
\begin{aligned}
x^{*}(k) & =\left(A x^{*}\right)(k)=b(k-1) e^{-\beta(k-1) x^{*}(k-1-\tau)}+\sum_{s=-\infty}^{k-2}\left(b(s) e^{-\beta(s) x^{*}(s-\tau)} W(k, s)\right) \\
& \leq \bar{b}+(1-\underline{a}) \sum_{s=-\infty}^{k-2} b(s) \leq \bar{b}+(1-\underline{a}) B^{+} .
\end{aligned}
$$

We also have

$$
\begin{aligned}
x^{*}(k) & =\left(A x^{*}\right)(k)=b(k-1) e^{-\beta(k-1) x^{*}(k-1-\tau)}+\sum_{s=-\infty}^{k-2}\left(b(s) e^{-\beta(s) x^{*}(s-\tau)} W(k, s)\right) \\
& \geq b(k-1) e^{-\beta(k-1)\left[\bar{b}+(1-\underline{a}) B^{+}\right]}+\sum_{s=-\infty}^{k-2}\left(b(s) e^{-\beta(s)\left[\bar{b}+(1-\underline{a}) B^{+}\right]} W(k, s)\right) \\
& \geq \underline{b} e^{-\bar{\beta}\left[\bar{b}+(1-\underline{a}) B^{+}\right]}+m \sum_{s=-\infty}^{k-2}\left(b(s) e^{-\bar{\beta}\left[\bar{b}+(1-\underline{a}) B^{+}\right]}\right) \\
& =\left(\underline{b}+m \sum_{s=-\infty}^{k-2} b(s)\right) e^{-\bar{\beta}\left[\bar{b}+(1-\underline{a}) B^{+}\right]} \geq\left(\underline{b}+m B^{-}\right) e^{-\bar{\beta}\left[\bar{b}+(1-\underline{a}) B^{+}\right]} .
\end{aligned}
$$

So, we get $\left(\underline{b}+m B^{-}\right) e^{-\bar{\beta}\left[\bar{b}+(1-\underline{a}) B^{+}\right]} \leq x^{*}(k) \leq \bar{b}+(1-\underline{a}) B^{+}$.

\section{Exponential stability of a positive almost periodic solution}

In this section, we study the exponential stability of a positive almost periodic solution.

Theorem 2 Assume that $\bar{\beta}\left[\bar{b}+(1-\underline{a}) B^{+}\right] \leq 1$ and $\underline{a}>\bar{\beta} \bar{b}$, then equation (1.2) has a unique exponentially stable almost periodic positive solution.

Proof Since $\bar{\beta}\left[\bar{b}+(1-\underline{a}) B^{+}\right] \leq 1$ holds, by Theorem 1 we know equation (1.2) has a unique almost periodic positive solution $x^{*}(k)$, and $\left(\underline{b}+m B^{-}\right) e^{-\bar{\beta}\left[\bar{b}+(1-\underline{a}) B^{+}\right]} \leq x^{*}(k) \leq \bar{b}+(1-\underline{a}) B^{+}$. Now we prove that $x^{*}(k)$ is exponentially stable.

Suppose that $x(k)$ is an arbitrary solution of equation (1.2) with the initial function $x(k)=$ $\phi(k)>0$ for $-\tau \leq k \leq 0$. Assume that the initial function of the almost periodic positive solution $x^{*}(k)$ is $x^{*}(k)=\psi(k)>0$ for $-\tau \leq k \leq 0$.

Consider the function $H(x)=\bar{\beta} \bar{b} e^{(\tau+1) x}-\underline{a} e^{x}+e^{x}-1, x \in[0,1]$.

Since $H(0)=\bar{\beta} \bar{b}-\underline{a}<0$, then there exists a constant $\mu \in(0,1)$ such that $H(\mu)<0$.

That is,

$$
-\underline{a} e^{\mu}+e^{\mu}+\bar{\beta} \bar{b} e^{(\tau+1) \mu}<1 .
$$


Let $y(k)=x(k)-x^{*}(k)$, we define $V(k)=|y(k)| e^{\mu k}$, then we get

$$
\begin{aligned}
\Delta V(k) & =V(k+1)-V(k)=|y(k+1)| e^{\mu(k+1)}-|y(k)| e^{\mu k} \\
& =|y(k+1)| e^{\mu(k+1)}-|y(k)| e^{\mu(k+1)}+|y(k)| e^{\mu(k+1)}-|y(k)| e^{\mu k} \\
& =(|y(k+1)|-|y(k)|) e^{\mu(k+1)}+\left(e^{\mu}-1\right)|y(k)| e^{\mu k} \\
& =\left(\left|x(k+1)-x^{*}(k+1)\right|-\left|x(k)-x^{*}(k)\right|\right) e^{\mu(k+1)}+\left(e^{\mu}-1\right)|y(k)| e^{\mu k} .
\end{aligned}
$$

Notice that

$$
\left|x(k+1)-x^{*}(k+1)\right|-\left|x(k)-x^{*}(k)\right| \leq-a(k)\left|x(k)-x^{*}(k)\right|+b(k)\left|e^{-\beta(k) x(k-\tau)}-e^{-\beta(k) x^{*}(k-\tau)}\right|,
$$

which, together with (4.2), leads to

$$
\begin{aligned}
\Delta V(k)= & V(k+1)-V(k) \\
\leq & \left(-a(k)\left|x(k)-x^{*}(k)\right|+b(k)\left|e^{-\beta(k) x(k-\tau)}-e^{-\beta(k) x^{*}(k-\tau)}\right|\right) e^{\mu(k+1)} \\
& +\left(e^{\mu}-1\right)|y(k)| e^{\mu k} \\
= & \left(-a(k) e^{\mu}+e^{\mu}-1\right)|y(k)| e^{\mu k}+b(k) e^{\mu}\left|e^{-\beta(k) x(k-\tau)}-e^{-\beta(k) x^{*}(k-\tau)}\right| e^{\mu k} \\
= & \left(-a(k) e^{\mu}+e^{\mu}-1\right) V(k)+b(k) e^{\mu}\left|e^{-\beta(k) x(k-\tau)}-e^{-\beta(k) x^{*}(k-\tau)}\right| e^{\mu k} .
\end{aligned}
$$

Let $M=\bar{b}+(1-\underline{a}) B^{+}+\max _{-\tau \leq k \leq 0, k \in Z}|\phi(k)-\psi(k)|$.

For $\forall k \in[-\tau, 0]$,

$$
\begin{aligned}
V(k) & =|y(k)| e^{\mu k} \leq|y(k)|=\left|x(k)-x^{*}(k)\right|=|\phi(k)-\psi(k)| \\
& \leq \max _{-\tau \leq k \leq 0, k \in Z}|\phi(k)-\psi(k)|<\bar{b}+(1-\underline{a}) B^{+}+\max _{-\tau \leq k \leq 0, k \in Z}|\phi(k)-\psi(k)|=M .
\end{aligned}
$$

We claim that

$$
V(k)<M \text { for all } k>0, k \in Z \text {. }
$$

Suppose that claim (4.4) is not true, then there must exist $K^{*} \in Z^{+}$such that $V\left(K^{*}\right) \geq M$ and $V(k)<M$ for $-\tau \leq k<K^{*}, k \in Z$.

It follows from (4.3) that

$$
\begin{aligned}
V\left(K^{*}\right)-V\left(K^{*}-1\right) \leq & \left(-a\left(K^{*}-1\right) e^{\mu}+e^{\mu}-1\right) V\left(K^{*}-1\right) \\
& +b\left(K^{*}-1\right) e^{\mu}\left|e^{-\beta\left(K^{*}-1\right) x\left(K^{*}-1-\tau\right)}-e^{-\beta\left(K^{*}-1\right) x^{*}\left(K^{*}-1-\tau\right)}\right| e^{\mu\left(K^{*}-1\right)} \\
\leq & \left(-\underline{a} e^{\mu}+e^{\mu}-1\right) V\left(K^{*}-1\right) \\
& +\bar{b} e^{\mu}\left|e^{-\beta\left(K^{*}-1\right) x\left(K^{*}-1-\tau\right)}-e^{-\beta\left(K^{*}-1\right) x^{*}\left(K^{*}-1-\tau\right)}\right| e^{\mu\left(K^{*}-1\right)}
\end{aligned}
$$


By the mean value theorem, we have

$$
\begin{aligned}
& \left|e^{-\beta\left(K^{*}-1\right) x\left(K^{*}-1-\tau\right)}-e^{-\beta\left(K^{*}-1\right) x^{*}\left(K^{*}-1-\tau\right)}\right| \\
& \quad=\left|-e^{-\xi}\left(\beta\left(K^{*}-1\right) x\left(K^{*}-1-\tau\right)-\beta\left(K^{*}-1\right) x^{*}\left(K^{*}-1-\tau\right)\right)\right| \\
& \quad \leq\left|\beta\left(K^{*}-1\right)\left(x\left(K^{*}-1-\tau\right)-x^{*}\left(K^{*}-1-\tau\right)\right)\right| \\
& \quad \leq \bar{\beta}\left|x\left(K^{*}-1-\tau\right)-x^{*}\left(K^{*}-1-\tau\right)\right|,
\end{aligned}
$$

in which $\xi$ lies between $\beta\left(K^{*}-1\right) x\left(K^{*}-1-\tau\right)$ and $\beta\left(K^{*}-1\right) x^{*}\left(K^{*}-1-\tau\right)$.

Thus, from (4.5) and (4.6), we get

$$
\begin{aligned}
V & \left(K^{*}\right)-V\left(K^{*}-1\right) \\
& \leq\left(-\underline{a} e^{\mu}+e^{\mu}-1\right) V\left(K^{*}-1\right)+\bar{b} e^{\mu} \bar{\beta}\left|x\left(K^{*}-1-\tau\right)-x^{*}\left(K^{*}-1-\tau\right)\right| e^{\mu\left(K^{*}-1\right)} \\
& =\left(-\underline{a} e^{\mu}+e^{\mu}-1\right) V\left(K^{*}-1\right)+\bar{b} e^{\mu} \bar{\beta}\left|x\left(K^{*}-1-\tau\right)-x^{*}\left(K^{*}-1-\tau\right)\right| e^{\mu\left(K^{*}-1-\tau\right)} e^{\mu \tau} \\
& =\left(-\underline{a} e^{\mu}+e^{\mu}-1\right) V\left(K^{*}-1\right)+\bar{b} e^{\mu} \bar{\beta}\left|y\left(K^{*}-1-\tau\right)\right| e^{\mu\left(K^{*}-1-\tau\right)} e^{\mu \tau} \\
& =\left(-\underline{a} e^{\mu}+e^{\mu}-1\right) V\left(K^{*}-1\right)+\bar{b} e^{\mu} \bar{\beta} V\left(K^{*}-1-\tau\right) e^{\mu \tau} .
\end{aligned}
$$

From (4.7), we obtain

$$
\begin{aligned}
V\left(K^{*}\right) & \leq\left(-\underline{a} e^{\mu}+e^{\mu}\right) V\left(K^{*}-1\right)+\bar{b} e^{\mu} \bar{\beta} V\left(K^{*}-1-\tau\right) e^{\mu \tau} \\
& <\left(-\underline{a} e^{\mu}+e^{\mu}\right) M+\bar{b} e^{\mu} \bar{\beta} M e^{\mu \tau} \\
& =\left(-\underline{a} e^{\mu}+e^{\mu}+\bar{\beta} \bar{b} e^{\mu(\tau+1)}\right) M<M,
\end{aligned}
$$

which contradicts $V\left(K^{*}\right) \geq M$.

So claim (4.4) is true. Hence $V(k)=|y(k)| e^{\mu k}<M$ for all $k>0, k \in Z$.

That is, $\left|x(k)-x^{*}(k)\right|<M e^{-\mu k}$ for all $k>0, k \in Z$, which means that $x^{*}(k)$ is exponentially stable.

The proof of Theorem 2 is complete.

\section{Competing interests}

The author declares that they have no competing interests.

\section{Author's contributions}

The author is entirely responsible for this research. The author read and approved the final manuscript.

\section{Acknowledgements}

The author thanks the referees for their valuable comments and suggestions in improving the presentation of the manuscript. This work is supported by Natural Science Foundation of Education Department of Anhui Province (KJ2014A043).

\section{Received: 6 January 2014 Accepted: 2 July 2014 Published: 04 Aug 2014}

\section{References}

1. Wazewska-Czyzewska, M, Lasota, A: Mathematical problems of the dynamics of red blood cells system. Rocz. Pol. Tow. Mat., 3 Mat. Stosow. 6, 23-40 (1976)

2. Kulenovic, MRS, Ladas, G: Linearized oscillations in population dynamics. Bull. Math. Biol. 49, 615-627 (1987)

3. Graef, JR, Qian, C, Spikes, PW: Oscillation and global attractivity in a periodic delay equation. Can. Math. Bull. 38, 275-283 (1996)

4. Kulenovic, MRS, Ladas, G, Sficas, YG: Global attractivity in population dynamics. Comput. Math. Appl. 18, 925-928 (1989) 
5. Xu, W, Li, J: Global attractivity of the model for the survival of red blood cells with several delays. Ann. Differ. Equ. 14(2), 357-363 (1998)

6. Jiang, DQ: Existence of positive periodic solutions for non-autonomous delay differential equations. Chin. Ann. Math. Ser. A 20(6), 715-720 (1999)

7. Li, JW, Wang, ZC: Existence and global attractivity of positive periodic solutions of a survival model of red blood cells. Comput. Math. Appl. 50(1-2), 41-47 (2005)

8. Agarwal, RP: Difference Equations and Inequalities: Theory, Methods and Applications, 2nd edn. Monographs and Textbooks in Pure and Applied Mathematics, vol. 228. Dekker, New York (2000)

9. Agarwal, RP, Wong, PJY: Advanced Topics in Difference Equations. Kluwer Academic, Dordrecht (1997)

10. Elayadi, S: An Introduction to Difference Equations, 3rd edn. Springer, New York (2005)

11. Fan, M, Wang, K: Global existence of positive periodic solutions of periodic predator-prey system with infinite delays. J. Math. Anal. Appl. 262(1), 1-11 (2001)

12. Ye, D, Fan, M: Periodicity in mutualism systems with impulse. Taiwan. J. Math. 10(3), 723-737 (2006)

13. Li, WT, Huo, HF: Existence and global attractivity of positive periodic solutions of functional differential equations with impulses. Nonlinear Anal. 59(6), 857-877 (2004)

14. Ma, MJ, Yu, JS: Existence of multiple positive periodic solutions for nonlinear functional difference equations. J. Math. Anal. Appl. 305(2), 483-490 (2005)

15. Fink, A: Almost Periodic Differential Equations. Lecture Notes in Mathematics, vol. 377. Springer, Berlin (1974)

16. He, CY: Almost Periodic Differential Equations. Higher Education Press, Beijing (1992)

17. Yuan, R: On almost periodic solutions of logistic delay differential equations with almost periodic time dependence. J. Math. Anal. Appl. 330, 780-798 (2007)

18. Ahmad, S, Stamov, GT: Almost periodic solutions of $n$-dimensional impulsive competitive systems. Nonlinear Anal., Real World Appl. 10(3), 1846-1853 (2009)

19. Abbas, S, Bahuguna, D: Almost periodic solutions of neutral functional differential equations. Comput. Math. Appl. 55(11), 2539-2601 (2008)

20. Geng, J, Xia, Y: Almost periodic solutions of a nonlinear ecological model. Commun. Nonlinear Sci. Numer. Simul. 16(6), 2575-2597 (2011)

21. Cheban, D, Mammana, C: Invariant manifolds, global attractors and almost periodic solutions of nonautonomous difference equations. Nonlinear Anal. 56(4), 465-484 (2004)

22. Guo, D: Nonlinear Functional Analysis. Shandong Science and Technology Press, Jinan (2001)

10.1186/1687-1847-2014-206

Cite this article as: Yao: Existence and exponential stability of the unique positive almost periodic solution for the Lasota-Wazewska difference model. Advances in Difference Equations 2014, 2014:206

\section{Submit your manuscript to a SpringerOpen ${ }^{\ominus}$ journal and benefit from:}

- Convenient online submission

- Rigorous peer review

- Immediate publication on acceptance

- Open access: articles freely available online

- High visibility within the field

- Retaining the copyright to your article 